\title{
Size Dependent Thermopower in Mesoscopic AuFe Wires
}

\author{
C. Strunk, M. Henny, and C. Schönenberger \\ Institut für Physik, Universität Basel, Klingelbergstrasse 82, CH-4056 Basel, Switzerland \\ G. Neuttiens and C. Van Haesendonck \\ Laboratorium voor Vaste-Stoffysica en Magnetisme, Katholieke Universiteit Leuven, \\ Celestijnenlaan 200 D, B-3001 Leuven, Belgium
}

(Received 11 March 1998)

\begin{abstract}
We have combined electron heating experiments and noise thermometry to perform quantitative measurements of the thermopower in mesoscopic samples. This new measuring technique allows us to detect finite size effects in the thermopower of narrow AuFe wires with an $\mathrm{Fe}$ concentration ranging from 50 to $3000 \mathrm{ppm}$. The size effects emerge when reducing the width of the wires below $\simeq 300 \mathrm{~nm}$ and may be related to a spin-orbit induced magnetic anisotropy close to the wire surface. [S0031-9007(98)07290-1]
\end{abstract}

PACS numbers: 72.15.Jf, 73.23.-b, 75.50.Lk

The scattering of conduction electrons at transition metal impurity spins (e.g., $\mathrm{Fe}, \mathrm{Cr}$, and $\mathrm{Mn}$ ) substantially alters the low temperature properties of noble metals (e.g., Au, $\mathrm{Ag}$, and $\mathrm{Cu})[1]$. For small concentrations $(\sim 100 \mathrm{ppm})$ of the magnetic dopant the Kondo effect gives rise to a logarithmic increase of the resistivity $\rho(T)$. Below the Kondo temperature $T_{K}$ a compensation cloud of conduction electrons is formed around the impurity spins which gradually cancels their magnetic moment. At higher concentrations ( $\sim 1$ at. \%) the Ruderman-Kittel-Kasuya-Yosida (RKKY) interaction between the randomly distributed impurity spins competes with the Kondo effect and causes a freezing of the impurity spins into a disordered configuration called a spin glass. This is reflected by the appearance of a typical broad maximum in $\rho(T)$ since the spin scattering rate again decreases at the lowest temperatures due to the freezing process.

Recent experimental work has addressed the existence of intrinsic length scales for both the Kondo effect and the spin glass freezing process [2-6]. The results have so far been controversial: While some groups [2,3] report a pronounced depression of the Kondo slope with decreasing film thickness and wire width, other authors claim that the observed size effects are small and can moreover be explained quantitatively in terms of disorder enhanced electron-electron interaction effects [5,6]. It was also noted that structural disorder may have an important influence on the spin dependent part of $\rho(T)$ for Kondo alloys [7] as well as for more concentrated spin glass alloys where a damping of the RKKY interaction occurs $[6,8]$.

Theoretical calculations have shown that in the single impurity limit spin-orbit interactions can induce a size dependent magnetic anisotropy which results in a size dependent resistivity [9]. For more disordered samples, an interplay between weak localization and the Kondo effect has been predicted which can account for both a disorder effect and a size effect [10]. In order to address the open questions related to the size effects, it is obviously interesting to look at other transport properties which are affected by the spin scattering. An excellent candidate is the thermoelectric power (TEP), $S(T)$, which according to Mott's rule directly reflects the pronounced energy dependence of the scattering time induced by the impurity spins [11]. Hence, the TEP is strongly enhanced in dilute magnetic alloys and should be even more sensitive to size effects than the resistivity. While in bulk $\mathrm{Au} S(T)$ is positive and very small, it switches sign upon adding Fe impurities and its absolute value can be as high as $15 \mu \mathrm{V} / \mathrm{K}$ [11]. Further increase of the $\mathrm{Fe}$ concentration into the spin glass regime again reduces $|S(T)|$ [12].

Up to now, reliable measurements of $S(T)$ could not be performed for mesoscopic samples. In this Letter, we present the first quantitative measurements of $S(T)$ in mesoscopic $\mathrm{AuFe}$ wires. Our measuring technique is based on current induced electron heating and noise thermometry for a direct measurement of the electron temperature. We observe a clear reduction of $|S(T)|$ when the width of the AuFe wires is reduced from 300 down to $100 \mathrm{~nm}$. Our measurements allow us to directly test the theoretical models which link the size dependence to a surface induced magnetic anisotropy [9]. Previous experiments on mesoscopic AuFe wires revealed a pronounced asymmetry of the differential resistance as a function of the heating current [13] which is absent in pure $\mathrm{Au}$ and can be traced back to the enhanced thermoelectric power of the AuFe [14]. However, the functional dependence of the TEP on size and temperature could not be determined so far.

The samples consist of pairs of AuFe wires of different width (forming thermocouples) connected at one end to a meandric wire which serves as a heater with resistance $R_{H}$ (see Fig. 1). The electron temperature $T_{H}$ in the heater is raised above the substrate temperature $T_{S}$ when a $d c$ current $I$ flows through the heater. The other ends of the thermocouple wires are connected to large contact pads which are assumed to remain at $T_{S}$. 


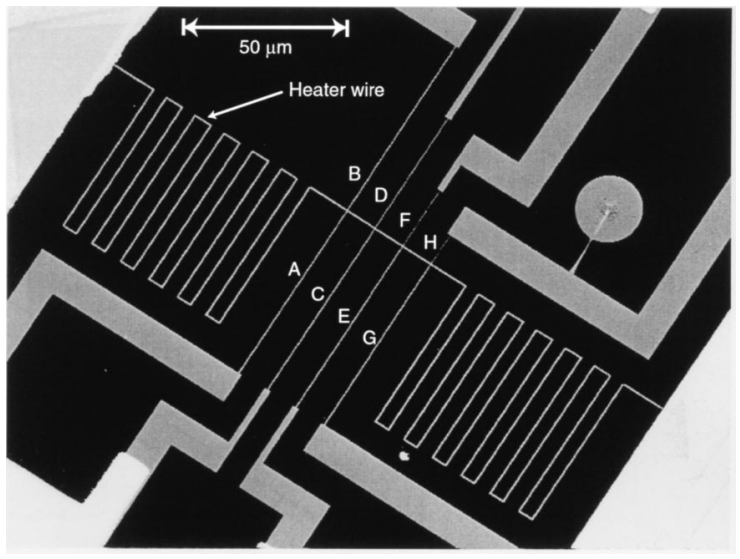

FIG. 1. Scanning electron micrograph of a typical sample. The four different thermocouples are labeled $A B, C D, E F$, and $G H$.

The wires $A, C, E$, and $G$ on one side of the $510 \mathrm{~nm}$ wide heater have the same nominal width of $300 \pm 15 \mathrm{~nm}$ and serve as a reference to detect small changes of the thermopower when varying the width of the wires labeled $B, D, F$, and $H$ on the other side. The latter wires have a width $w$ of $305,220,140$, and $105 \mathrm{~nm}$, respectively, and their length increases with increasing $w$ to keep the thermal conductance of all wires constant. The length of the narrowest wires as well as the distance between the junctions is $10 \mu \mathrm{m}$ while the total length of the meandric heater is $1.4 \mathrm{~mm}$. This geometry provides a nearly flat profile of $T_{H}$ along the heater except at both ends of the heater close to the large contact pads [15]. We note thatregardless of the temperature profile in the sample - in the absence of a size effect in $S(T)$ no thermovoltages are expected to develop across our mesoscopic thermocouples made entirely from AuFe.

The samples have been prepared by electron beam lithography and evaporation of $99.999 \%$ pure Au. In a second step $\mathrm{Fe}$ ions have been implanted at several energies to provide a reasonably constant doping profile perpendicular to the film [5]. Two series of samples were prepared having nominal Fe concentrations of 50 and $3000 \mathrm{ppm}$, respectively. Prior to implantation, the $30 \mathrm{~nm}$ thick films had a sheet resistance $R_{\square}$ of $0.3 \Omega$ at $4.2 \mathrm{~K}$. After implantation $R_{\square}$ of the $50 \mathrm{ppm}$ Kondo samples remained unchanged while $R_{\square}$ of the 3000 ppm spin glass samples increased to $0.7 \Omega$. This corresponds to an elastic mean free path $l_{\mathrm{e} 1}$ of 90 and $40 \mathrm{~nm}$, respectively. Scanning electron microscopy and atomic force microscopy images indicate that the films are polycrystalline with a grain size (20-30 nm) considerably smaller than the wire width. We emphasize that the size dependence, which we will report in this Letter, cannot be explained by a simple disorder effect since all wires are prepared simultaneously, resulting in a value of $l_{\mathrm{el}}$ which is independent of the wire width. Most of the measurements have been performed in a ${ }^{3} \mathrm{He}$ cryostat at a bath temperature of $300 \mathrm{mK}$.

The idea of our thermopower experiment is the following: If a current $I$ is sent through the heater wire the elec- tron temperature in the heater rises above the substrate temperature $T_{S}$. The temperature at the thermocouple junctions $T_{J}$ will be slightly lower than the average temperature of the heater $T_{H}$ because of the finite thermal conductance of the thermocouple wires. The resulting thermoelectric voltage across the thermocouples will be symmetric in $I$ since $T_{J}(I)$ is symmetric in $I$ :

$$
V_{\mathrm{th}}(I)=\int_{T_{S}(I)}^{T_{J}(I)} \Delta S(T) d T,
$$

where $\Delta S(T)=S_{\text {wide }}(T)-S_{\text {narrow }}(T)$ is the thermopower difference between the wide and narrow wire of the thermocouple. In order to increase the sensitivity of our measurements, we measure the differential resistance $d V / d I$ rather than $V(I)$ across our thermocouples. Since $V_{\mathrm{th}}(I)$ is symmetric in $I, d V / d I$ will predominantly be antisymmetric in $I$. According to Eq. (1)), the antisymmetric part of $d V / d I$ is directly linked to $\Delta S(T)$ :

$$
\frac{d V_{\mathrm{th}}}{d I}=\Delta S\left(T_{J}\right) \frac{d T_{J}}{d I}-\Delta S\left(T_{S}\right) \frac{d T_{S}}{d I} .
$$

The second term in Eq. (2) represents the contribution of the also elevated substrate temperature $T_{S}$ at higher currents. To extract $\Delta S(T)$, Eq. (2) has to be solved self-consistently. Similar cross-shaped geometries have been used to measure mesoscopic TEP fluctuations in GaAs quantum wires [16] and the TEP of quantum point contacts [17]. However, in these experiments the electron temperature has not been measured independently.

The average temperature in the heater wire $T_{H}(I)$ has been determined by measuring the spectral density $S_{V}(I)=4 k_{B} T_{H}(I) R_{H}$ of the voltage fluctuations across the heater wire as a function of current bias [15]. For typical heater resistances $R_{H}$ of $1-2 \mathrm{k} \Omega$ the electron temperature could be determined with an accuracy of about $50 \mathrm{mK}$. Figure 2 shows an example of $T_{H}(I)$ for the $3000 \mathrm{ppm}$ sample together with a fit corresponding to the semiempirical form $T_{H}(I)=\left(a I^{2}+T_{0}^{b}\right)^{1 / b}$, where $T_{0}=0.3 \mathrm{~K}$ is the temperature of the sample stage. The parameters for the best fit were $a=0.0386 \mathrm{~K}^{b} / \mu \mathrm{A}^{2}$ and $b=4.1$. In agreement with previous electron heating experiments in this temperature range [18], we find that $b$ ranges from 4.0 to 4.3 for different samples. A detailed discussion of the physical origin of the exponent $b$ is beyond the scope of this paper and will be given elsewhere. With a heating current of $80 \mu \mathrm{A}$ electron temperatures up to $4 \mathrm{~K}$ have been achieved.

The temperature at the junction $T_{J}(I)$ has been determined by a numerical solution of the heat diffusion equation based on Ref. [19]. The calculation uses the measured $T_{H}(I)$ curve to take into account the cooling through the electron-phonon scattering and the result is indicated by the dotted line in Fig. 2. A comparison of the current dependence of the resistivity for the different sections of the heater wire confirms that the local reduction of $T_{H}$ remains smaller than $10 \%$. For the highest currents a 


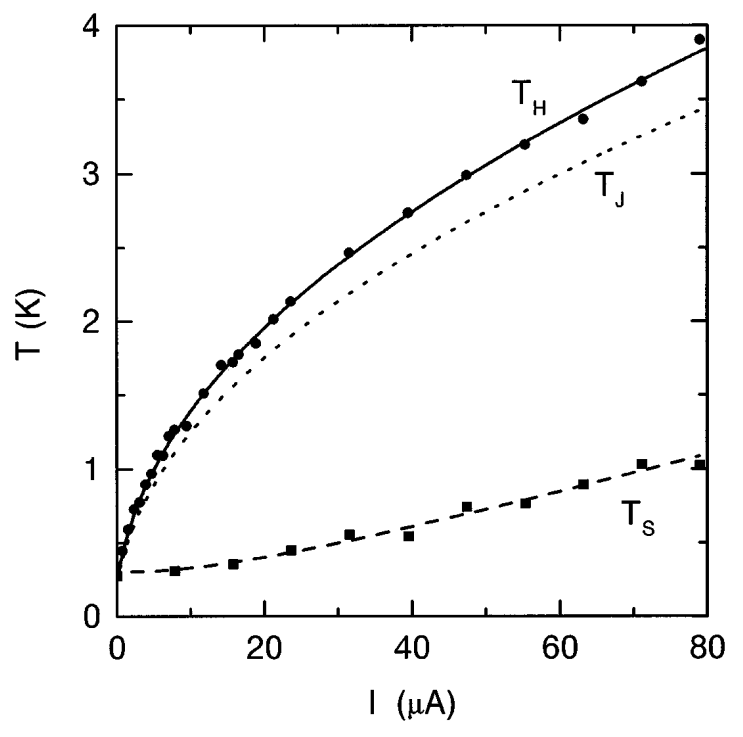

FIG. 2. Electron temperatures $T_{H}(I)$ of the heater and $T_{S}(I)$ of the substrate monitor wire together with fits as described in the text. The dotted line corresponds to a value of $a=$ $0.0247 \mathrm{~K}^{b} / \mu \mathrm{A}^{2}$ and indicates the temperature $T_{J}(I)$ at the thermocouple junction $G H$.

power of $\simeq 10 \mu \mathrm{W}$ is dissipated in the heater, which is sufficient to also raise the substrate temperature up to $T_{S} \simeq$ $1 \mathrm{~K}$. The open symbols in Fig. 2 show $T_{S}$ measured on an independent Au wire patterned close to the AuFe sample while current is sent through the heater wire. The dashed line is a fit of the form $T_{S}=\sqrt{a^{\prime} I^{2}+T_{0}^{2}}$ with $a^{\prime}=$ $0.00017 \mathrm{~K}^{2} / \mu \mathrm{A}^{2}$. This functional dependence is expected since the thermal coupling between the sample stage of the cryostat and the substrate is metallic with a thermal conductance depending linearly on temperature.

The response of the thermocouples to the dc heating current is detected with a lock-in amplifier by superimposing a small ac current of $1 \mu \mathrm{A}$ and $116 \mathrm{~Hz}$. We first measured in detail the $d V / d I$ signal for the 50 ppm thermocouples. As shown in the inset in Fig. 3, the thermocouple $E F$ clearly reveals the presence of a signal which is antisymmetric in $I$ and is of the order of $1 \mathrm{~m} \Omega$. The antisymmetric signal increases when the difference in width $\Delta w$ increases, which can be linked to a decrease of the thermopower in the narrower AuFe wires. According to the Gorter-Nordheim rule [11] the measured TEP in our Kondo samples is strongly reduced by the nonmagnetic scattering. The reduction factor is given by the ratio of the total resistivity $\rho_{\text {tot }}$ and the resistivity contribution $\rho_{\mathrm{Fe}}$ [20] of the Fe impurities. Relying on the reported resistivity $\rho_{\text {Fe }}$ for bulk samples $[1,21]$, we estimate $\rho_{\text {tot }} / \rho_{\mathrm{Fe}} \simeq 12$. According to Eq. (2) and the available data for the thermopower in bulk Kondo alloys [12] we find that for the thermocouple $E F$ the observed thermopower signal $\Delta S(T)$ is of the order of $6 \%$ of the bulk thermopower. Unfortunately, the poor signal to noise ratio for our Kondo samples does not allow us to draw more quantitative conclusions concerning the width dependence of the thermopower.

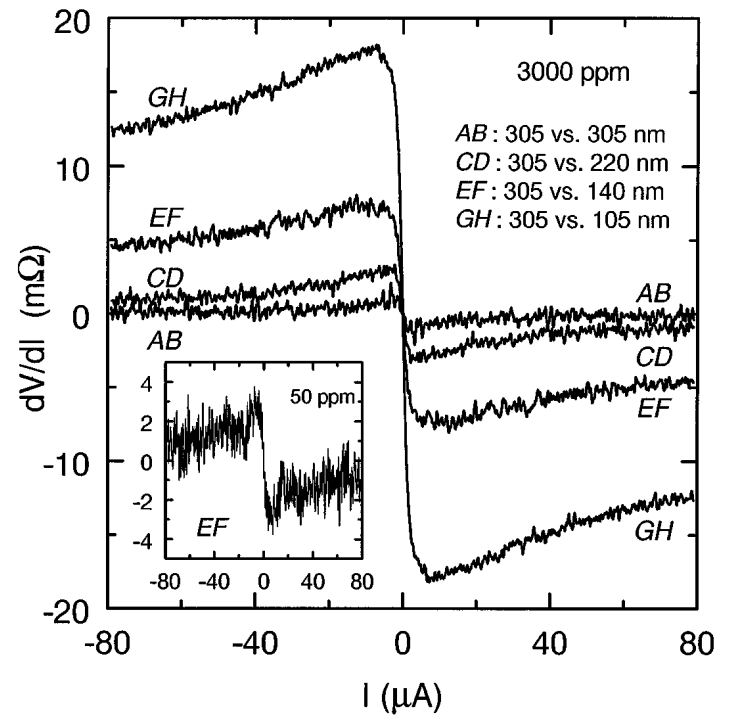

FIG. 3. Antisymmetric part of the differential resistance for the different thermocouples. Voltage contact $V_{+}$was connected to one of the reference wires $A, C, E$, and $G$ (nominal $w=300 \mathrm{~nm}$ ) while $V_{-}$was connected to one of the narrow wires $B, D, F$, and $H \quad(w=305,220,140$, and $105 \mathrm{~nm}$, respectively). Trace $A B$ is an average of several $300 / 300 \mathrm{~nm}$ combinations. Inset: Asymmetric part of $d V / d I$ for thermocouple $E F$ of a sample with 50 ppm Fe.

For the $3000 \mathrm{ppm}$ samples a comparison with the data for bulk alloys $[1,21]$ indicates that $\rho_{\mathrm{tot}} \simeq \rho_{\mathrm{Fe}}$ for our relatively clean samples. Consequently, the thermoelectric voltages are considerably larger than for the Kondo samples and a quantitative data analysis becomes possible. Figure 3 shows the antisymmetric part of the $d V / d I$ signal from the thermocouples $A B, C D, E F$, and $G H$, respectively (see Fig. 1). For the thermocouple $G H$ which has the largest difference in width $\Delta w, d V / d I$ rises very sharply from zero, shows a maximum around $7 \mu \mathrm{A}$, and slowly decreases for higher currents. For decreasing $\Delta w$ in the thermocouples $E F$ and $C D$ the asymmetry is systematically reduced while the overall shape of the $d V / d I$ signal remains similar. For the thermocouple $A B$, where $\Delta w$ is nominally zero, a residual small asymmetry is observed which is of random sign for different samples and can be attributed to small size differences related to imperfections of the lithographic patterning.

In Fig. 4 we have plotted the temperature dependence of $\Delta S(T)$ for the different 3000 ppm thermocouples according to Eq. (2). For the Kondo as well as for the spin glass samples $\Delta S(T)=S_{\text {wide }}-S_{\text {narrow }}$ is negative, implying that $|S(T)|$ becomes smaller when reducing the width of the wires. For the $3000 \mathrm{ppm}$ data (see Fig. 4) $\Delta S(T)$ displays a nearly linear variation above $1.5 \mathrm{~K}$ which is close to the spin glass freezing temperature $T_{f}$ for $3000 \mathrm{ppm}$ [1]. The inset in Fig. 4 shows $|d \Delta S(T) / d T|$ for the linear regime as a function of the width of the narrower wire of the thermocouples. At lower temperatures $|\Delta S(T)|$ decreases more rapidly and is nearly zero at $0.4 \mathrm{~K}$. The measured values of $|\Delta S(T)|$ range up to $0.8 \mu \mathrm{V} / \mathrm{K}$ which should 


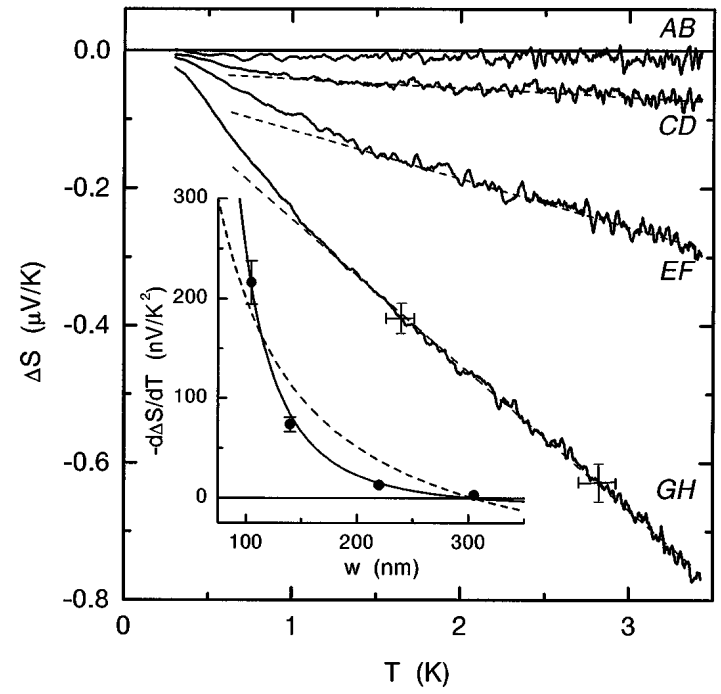

FIG. 4. Difference in thermopower as function of temperature for varying difference in wire width. The dashed lines illustrate the linear behavior ot $\Delta S(T)$ above $1.5 \mathrm{~K}$. The error bars indicate the uncertainty introduced by the correction of the temperature profile. Inset: Slope of $|\Delta S(T)|$ at $T=3 \mathrm{~K}$ as a function of the width of the narrow wire. The lines are best fits of a $1 / w^{3}$ (solid line) and a $1 / w$ (dashed line) dependence.

be compared with the value $|S(T)| \simeq 7 \mu \mathrm{V} / \mathrm{K}$ observed for bulk AuFe samples with a comparable Fe concentration [12]. On the other hand, the thermoelectric voltages become very small when both wires forming the thermocouples are wider than $300 \mathrm{~nm}$. We therefore conclude that for Kondo as well as for spin glass AuFe wires the thermopower is significantly reduced when reducing the width down to $100 \mathrm{~nm}$.

What is the origin of the size dependence? We have checked that the asymmetric $d V / d I$ is absent in undoped samples. For the more dilute alloys ( $\$ 500 \mathrm{ppm}$ ) a magnetic field of $17 \mathrm{~T}$ completely suppresses the asymmetry. This proves that the observed thermoelectric voltages are indeed related to the magnetic scattering. Since the size effects are also present in the Kondo samples, it is reasonable to link them to a single impurity effect along the lines of Refs. [9,10]. For relatively high temperatures $T \gtrsim T_{f}$, a considerable fraction of the magnetic impurity spins is still free to flip independently in the more concentrated spin glass wires and are therefore expected to be sensitive to the spin-orbit induced magnetic anisotropy proposed in Ref. [9]. As shown in the inset in Fig. 4, $|d \Delta S(T) / d T|$ increases more rapidly with decreasing width $\left(\propto 1 / w^{3}\right)$ for the $3000 \mathrm{ppm}$ thermocouples than the predicted $1 / w$ dependence of the slope of the Kondo resistivity [9]. At temperatures below $T_{f}$ the spin flip scattering by the individual magnetic moments is suppressed by the strong internal fields which are present in the spin glass phase. This is consistent with the vanishing of the $\Delta S(T)$ below $0.4 \mathrm{~K}$ (see Fig. 4).

In conclusion, we have developed a new technique for quantitative measurements of the thermoelectric power on a mesoscopic scale. Our measurements clearly reveal a size dependence of the thermoelectric power of mesoscopic AuFe wires when the width of the wires is reduced below $\simeq 300 \mathrm{~nm}$. Our observations can be understood in terms of the magnetic anisotropy which affects the spins close to the surface of the sample. The spin glass freezing at lower temperatures suppresses the size effects.

We are much indebted to Y. Bruynseraede, V. Chandrasekhar, J. Eom, J. Devreese, V. Fomin, V. Gladilin, and A. Zawadowski for stimulating discussions and to H. Pattyn, L. Lanz, and H. Birk for their help with the sample preparation and the measurement setup. The work at Basel has been supported by the Swiss National Science Foundation. The work at Leuven has been supported by the Fund for Scientific Research-Flanders (FWO) as well as by the Flemish Concerted Action (GOA) and the Belgian Inter-University Attraction Poles (IUAP).

[1] For a recent review, see, e.g., J. A. Mydosh, Spin Glasses, An Experimental Introduction (Taylor \& Francis, London, 1993).

[2] G. Chen and N. Giordano, Phys. Rev. Lett. 66, 209 (1991).

[3] J. F. DiTusa et al., Phys. Rev. Lett. 68, 678 (1992).

[4] K. R. Lane, M. Park, M. S. Isaacson, and J. M. Parpia, Phys. Rev. B 51, 945 (1995).

[5] V. Chandrasekhar et al., Phys. Rev. Lett. 72, 2053 (1994).

[6] G. Neuttiens et al., Europhys. Lett. 34, 617 (1996).

[7] M. A. Blachly and N. Giordano, Europhys. Lett. 27, 687 (1994).

[8] R. Buchmann, H.P. Falke, H.P. Jablonski, and E.F. Wassermann, Phys. Rev. B 17, 4315 (1978).

[9] O. Újsághy, A. Zawadowski, and B.L. Gyorffy, Phys. Rev. Lett. 76, 2378 (1996); O. Újsághy and A. Zawadowski, Phys. Rev. B 57, 11598 (1998); Phys. Rev. B 57, 11609 (1998); V. Fomin et al., Solid State Commun. 106, 293 (1998).

[10] I. Martin, Y. Wan, and P. Phillips, Phys. Rev. Lett. 78, 114 (1997).

[11] R. D. Barnard, Thermoelectricity in Metals and Alloys (Taylor \& Francis, London, 1972).

[12] D. K. C. MacDonald, W. B. Pearson, and I. M. Templeton, Proc. Roy. Soc. London A 266, 161 (1962).

[13] J. Eom et al., Phys. Rev. Lett. 77, 2276 (1996).

[14] G. Neuttiens et al., Europhys. Lett. 42, 185 (1998).

[15] M. Henny et al., Appl. Phys. Lett. 71, 773 (1997).

[16] B. L. Gallagher et al., Phys. Rev. Lett. 64, 2058 (1990).

[17] L. W. Molenkamp et al., Phys. Rev. Lett. 65, 1052 (1990).

[18] See, e.g., G. Bergmann, Wei Wei, Yao Zou, and R. M. Mueller, Phys. Rev. B 41, 7386 (1990); J. F. DiTusa et al., Phys. Rev. Lett. 68, 1156 (1992).

[19] K. E. Nagaev, Phys. Rev. B 52, 4740 (1995).

[20] Here we assume that the magnetic TEP dominates all other contributions, which is well justified for dilute magnetic alloys [11].

[21] O. Laborde and P. Radhakrishna, Solid State Commun. 9, 701 (1971). 